\title{
O DESENVOLVIMENTO DAS FUNÇÕES TRIGONOMÉTRICAS A PARTIR DE UMA ABORDAGEM HISTÓRICO-EPISTEMOLÓGICA
}

\section{THE DEVELOPMENT OF TRIGONOMETRIC FUNCTIONS FROM A HISTORICAL- EPISTEMOLOGICAL APPROACH}

\author{
Rebecca Lourenço \\ Universidade Estadual do Norte do Paraná - Campus Cornélio Procópio (UENP), \\ profrebeccalourenco@gmail.com \\ William Júnior do Nascimento \\ Universidade Federal do Paraná - Campus Avançado em Jandaia do Sul (UFPR), \\ williamjn@ufpr.br \\ Simone Luccas \\ Universidade Estadual do Norte do Paraná (UENP) - Campus Cornélio Procópio, \\ simoneluccas@uenp.edu.br
}

\begin{abstract}
Resumo
Este trabalho apresenta uma reconstrução histórico-epistemológica acerca das Funções Trigonométricas, com ênfase em demonstrar como se desenvolveu este conhecimento desde a antiguidade até seu estágio atual, de forma que possamos compreender o processo de construção do conhecimento e refletir sobre o mesmo. Quais povos e quais fatores levaram os antigos a desenvolverem este conhecimento, sua composição estrutural, articulação com demais conhecimentos, assim como sua funcionalidade para solucionar problemas são alguns dos fatores a serem considerados. Propõe-se, portanto, apresentar como se deu a origem do conhecimento Trigonométrico a partir dos babilônios e egípcios, ressaltar o processo de construção do conhecimento Trigonométrico, por meio de cálculos e figuras geométricas, de acordo com contribuições dos gregos, árabes e hindus, os quais proporcionaram subsídios para que, posteriormente, os europeus desenvolvessem o estudo das funções trigonométricas no ciclo trigonométrico e por meio de representações gráficas. Enfim, intenciona-se fomentar uma maior compreensão a cerca deste conhecimento matemático, a fim de proporcionar um conhecimento mais aprofundado e com significados.
\end{abstract}

Palavras-chave: História da Matemática; Trigonometria no Triângulo Retângulo; Funções Trigonométricas; Abordagem Histórico-Epistemológica. 


\begin{abstract}
This work presents a historical-epistemological reconstruction about Trigonometric Functions, with an emphasis on demonstrating how this knowledge has developed from antiquity to its present stage, so that we can understand the process of knowledge construction and reflect on it. What peoples and what factors led the ancients to develop this knowledge, its structural composition, articulation with other knowledge, as well as its functionality to solve problems are some of the factors to be considered. It is proposed, therefore, to present as the origin of the Trigonometric knowledge from the Babylonians and Egyptians, to emphasize the process of construction of the Trigonometric knowledge, by means of calculations and geometric figures, according to contributions of the Greeks, Arabs and Hindus, which provided subsidies for the later Europeans to develop the study of trigonometric functions in the trigonometric cycle and through graphical representations. Finally, it is intended to foster a greater understanding of this mathematical knowledge in order to provide a deeper understanding and meaning.
\end{abstract}

Keywords: History of Mathematics; Trigonometry in the Triangle Rectangle; Trigonometric Functions; Historical-Epistemological Approach.

\title{
Introdução
}

A História da Matemática vem se consolidando durante as últimas décadas como uma área de conhecimento e de investigação em Educação Matemática (LOPES, ALVES 2014). Ao considerá-la como metodologia de ensino e fazer uso em sala de aula, o professor pode adquirir ferramentas para mostrar o porquê de estudar determinados conteúdos, podendo promover aulas mais dinâmicas e interessantes (LOPES, FERREIRA, 2013).

De acordo com D’Ambrosio (1999), as práticas educativas se fundam na cultura, estilos de aprendizagem e tradições, ao passo que a história compreende registro desses fundamentos. Portanto, é difícil compreender a Matemática sem estudar sua história, cujas raízes estão diretamente ligadas à História da humanidade.

Desvincular a matemática das outras atividades humanas é um dos maiores erros que se pratica particularmente na educação da matemática. Em toda a evolução da humanidade, as ideias matemáticas vêm definindo estratégia de ação para lidar com o ambiente, criando e desenhando instrumento para esse fim e buscando explicações sobre os fatos e fenômenos da natureza e para própria existência (D'AMBRÓSIO, 1999, p.97).

A própria História da Matemática nos revela que essa Ciência foi desenvolvida a partir de problemas de ordem prática, intimamente ligado à cultura dos povos (MELO, FILEMAN, 2016). Seu desenvolvimento se deu por meio de problemas relativos às necessidades e preocupações de povos, com finalidade de responder questões de diferentes origens e contextos, revelando a Matemática como uma criação humana que 
estabelece relações com outras áreas do conhecimento como: música, acústica, eletricidade e mecânica (OLIVEIRA, 2013).

Ao citar a História da Matemática em sua obra "Três estudos sobre história e educação matemática”, Miguel (1993) lista várias contribuições que podem ser obtidas por meio da História da Matemática, dentre as quais enfatizamos a abordagem históricoepistemológica, a qual pode ser caracterizada como um instrumento revelador da natureza da Matemática. Neste sentido, Lucas (2010. p. 27) destaca que "análises epistemológicas corretas de conceitos, no domínio do ensino de Ciências, podem ajudar na transposição de barreiras da contradição e da falta de significado que podem levar muitos estudantes ao não entendimento de assuntos científicos". Alves e Tatsch (2017) complementam afirmando que o aluno precisa ser ativo e construtor de suas ideias e de seu conhecimento, precisa reconhecer o desenvolvimento histórico do conhecimento científico e sua importância para seu próprio crescimento pessoal e da sociedade em que vive.

Portanto, fundamentada na História da Matemática e na Epistemologia, este trabalho apresenta uma reconstrução histórico-epistemológica acerca das Funções Trigonométricas, com ênfase em demonstrar como se desenvolveu este conhecimento desde a antiguidade até seu estágio atual, de forma que possamos compreender o processo de construção do conhecimento e refletir sobre o mesmo. De acordo que este trabalho constituiu uma parte da dissertação "Funções Trigonométricas: produção de uma sequência didática potencialmente significativa à luz da abordagem históricoepistemológica" produzida pelos mesmos autores.

\section{O início da Trigonometria}

A Trigonometria esteve presente no desenvolvimento matemático de diversos povos desde os primórdios das civilizações mais antigas. O termo Trigonometria surgiu somente em 1595, pelo matemático, astrônomo e teólogo Pitiscus (1561 - 1613), mas historicamente, por vários séculos, seus estudos estavam inseridos na área de Geometria, área de estudo que se desenvolveu de acordo com necessidades práticas, utilizada também para contar, medir e desenhar (BOYER, 2012; BERLINGHOFF, GOUVÊA, 2012).

Estudos trigonométricos tiveram seus primeiros indícios registrados em rudimentos históricos tanto no Egito quanto na Babilônia datados 3000 a.C. e na China em aproximadamente 1110 a.C. (MENDES, 1997; COSTA, 1997). Obras importantes como o papiro Cairo (3000 a.C.) e papiro Rhind (1650 a.C.) evidenciam que os antigos já possuíam conhecimento a respeito dos ângulos, relações trigonométricas e triângulos retângulos, de modo que os aplicavam em diversos contextos como: construção de pirâmides, medição de sombras do gnômon (relógio do Sol) para determinar horas do dia, cobranças de impostos para plantio de terras férteis nas margens de rios, divisão de terras, cálculos astronômicos, entre outros (EVES, 2011).

Seguindo os egípcios, no Oriente Antigo, os gregos contribuíram para um desenvolvimento significativo da Matemática e da Trigonometria. Diante do racionalismo crescente, muito dos conhecimentos se desenvolveram de forma a responder questões 
do tipo "como" e "por quê", tendo em vista que a visão estática das coisas se tornou insustentável (BOYER, 2012). A Grécia foi berço de muitos conhecimentos a respeito da trigonometria, lugar em que diversos pensadores marcaram significativamente a História da Matemática, tais como: Tales e Pitágoras, ambos por volta do século VI a.c..

Dentre os conhecimentos desenvolvidos por esses pensadores gregos, destacamse a sistematização referente à semelhança de triângulos definida a partir do Teorema de Tales e a relação entre lados de um triângulo retângulo consolidado por Pitágoras e conhecida como Teorema de Pitágoras. Contudo, de acordo com o historiador Boyer (2012), relatos históricos sugerem que tais conhecimentos já eram de domínio dos babilônios e dos egípcios, marcando historicamente o início da Trigonometria com forte caráter geométrico.

\section{O desenvolvimento das relações seno e cosseno}

\section{A geometria das tabelas de cordas}

Historicamente, a Matemática ficou marcada por ser muito utilizada em cálculos na Astronomia. Consequentemente, as relações seno e cosseno foram desenvolvidas concomitantemente em um contexto astronômico baseado em estudos geométricos. Os gregos dedicavam-se a estes estudos, pois queriam compreender como a Terra e o Sol se moviam, distâncias entre Terra, Sol e Lua, entre outros. Para isto, utilizavam de métodos geométricos, como as tabelas de cordas, também conhecidas como tabelas trigonométricas (PEREIRA, MOREY, 2015).

Neste contexto, o termo corda representa o segmento de reta que une dois pontos extremos de um arco do círculo (KENNEDY, 1992), conforme representado na Figura 1 pelo segmento $\overline{R F}$.

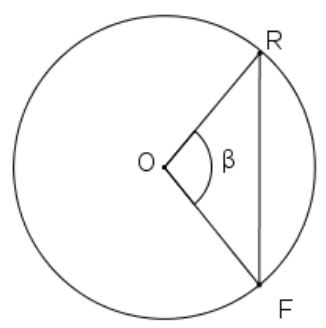

Figura 1 - Representação do comprimento da corda no círculo.

Fonte: Adaptado de Boyer (2012)

Os cálculos sobre cordas eram obtidos por meio de círculos, os quais eram considerados objetos de estudo desde a antiguidade. Para isto, Hiparco (190 - 120 a.C.) generalizou o trabalho de Hipsicles (240 - 170 a.C.) sobre a divisão do zodíaco em 360 partes para a divisão em 360 partes de qualquer círculo, sendo ele o primeiro grego a utilizar unidade de medida graus (NASCIMENTO, 2005).

A primeira tabela de cordas feita por Hiparco, por volta de 150 a.C., tratava do cálculo de comprimento de cordas de um arco de círculo arbitrário (PEREIRA, MOREY, 2015). Posteriormente, Claudio Ptolomeu (127 - 150 d.C.) realizou um importante trabalho para a Astronomia denominado "Syntaxis Mathemática" (Coleção matemática). Anos mais 
tarde, este trabalho foi considerado pelos árabes como a maior obra já existente até a época, ficando conhecida como "Almagesto" (Al Magest = A maior), a qual possuía a primeira tabela trigonométrica que chegou até os dias atuais (EVES, 2011; PEREIRA, MOREY 2015).

No Almagesto, Ptolomeu se baseou em métodos geométricos considerando o círculo e seus elementos, e assim construiu a tabelas de cordas a fim de facilitar os cálculos astronômicos. Baseado em Hiparco, dividiu em 360 partes o círculo, representado atualmente por $360^{\circ}$ (trezentos e sessenta graus), o diâmetro em 120 partes e cada parte em 60 porções, representado atualmente por 60' (sessenta minutos) e as 60 porções dividiu novamente por 60, representado atualmente por 60" (sessenta segundos), considerando o raio igual a 60. Essas divisões podem se justificar pelo fato dos gregos utilizarem a base sexagesimal dos babilônios (KENNEDY, 1992; KLINE, 1972; PEREIRA, MOREY, 2015).

Para a construção das tabelas de cordas, Ptolomeu considerou um círculo e relacionou o arco central com o comprimento da corda. Utilizando-se da geometria euclidiana e com base em um raio de 60 unidades, calculou o comprimento das cordas representado no Almagesto por crd (segmento $\overline{R F}$ da Figura 1).

Pereira e Morey (2015) relatam como Ptolomeu construiu as tabelas de cordas:

Para a construção dessas tabelas, Ptolomeu utilizou o cálculo das cordas de alguns ângulos, identificando-as por meio dos lados de polígonos regulares inscritos, como, por exemplo: $c r d 36^{\circ}$ é o lado de um decágono inscrito; $\operatorname{crd} 60^{\circ}$ é o lado do hexágono inscrito; $\operatorname{crd} 72^{\circ}$ é o lado do pentágono inscrito; $c r d 90^{\circ}$ é o lado de um quadrado inscrito; $c r d 120^{\circ}$ é o lado do triangulo equilátero inscrito. Para isso, ele apresenta a construção desses polígonos e a determinação do comprimento do lado desses polígonos (PEREIRA, MOREY, 2015. p. 145).

Tomando como exemplo o $\mathrm{crd} 90^{\circ}$, constrói-se um quadrado $A B C D$ inscrito no círculo, como representado na Figura 2.

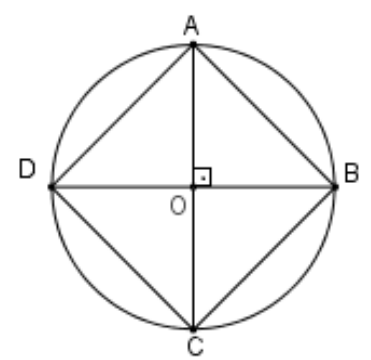

Figura 2 - Comprimento da corda de um ângulo de $90^{\circ}$.

Fonte: Os autores (2018).

$\mathrm{Na}$ Figura 2, observa-se que o quadrado $A B C D$ corresponde a quatro triângulos retângulos inscritos. Para encontrar o comprimento da corda basta considerar um triângulo retângulo e aplicar o teorema de Pitágoras para determinar o lado que representa o comprimento da corda. No triângulo $A O B$ os segmentos $\overline{A O}$ e $\overline{B O}$ são os 
raios do círculo representado por $r$ e o segmento $\overline{A B}$ o comprimento da corda que se deseja encontrar (MENDES, 1997). Aplicando o teorema de Pitágoras encontra-se o comprimento da corda $\overline{A B}$ :

$$
\begin{aligned}
& \text { hipotenusa }{ }^{2}=\text { cateto }^{2}+\text { cateto }^{2} \\
& \overline{A B}^{2}=\overline{A 0}^{2}+{\dot{B O^{2}}}^{2} \\
& \left(\text { crd } 90^{\circ}\right)^{2}=r^{2}+r^{2} \\
& \text { crd } 90^{\circ}=r \sqrt{2}
\end{aligned}
$$

Como Ptolomeu considerava raio igual a 60 unidades, o valor numérico para crd $90^{\circ}$ encontrado na tabela de cordas elaborada por ele é de 84 $32^{\prime} 03^{\prime \prime}$ (BERLINGHOFF, GOUVÊA, 2012; SCOTT,1969), número este representado na notação sexagesimal. Ao passar para o sistema decimal esse número é representado por $84+32 / 60+03 / 60$, que corresponde a 84,58.

Para calcular outros ângulos, Ptolomeu utilizou de deduções geométricas a partir de cálculos sobre a corda e soma de dois arcos: a corda do arco-metade e a corda da diferença de dois arcos, utilizando para isto além do símbolo $\mathrm{crd}$, o símbolo $\overline{c r d}$ que representava comprimento da corda de arco complementar (MENDES, 1997).

Historicamente, as tabelas de cordas feitas por Ptolomeu presentes no Almagesto foram consideradas uma aproximação das tabelas de senos e serviram de base para quase toda astronomia posicional até os trabalhos de Copérnico (1473 - 1543) (PEREIRA, MOREY 2015; KENNEDY, 1992; BERLINGHOFF, GOUVÊA, 2012). Neste sentido, os gregos construíram tabelas de cordas que relacionavam apenas conceitos geométricos dos círculos, como arcos e cordas. Posteriormente, tais tabelas passaram por modificações diante contribuições de diversos matemáticos e astrônomos, até chegar à Trigonometria que conhecemos atualmente (GOMES, 2011).

\section{Meia corda: seno}

Apesar de toda a contribuição da civilização grega, documentos relatam que a mais antiga tabela de senos foi descoberta na Índia entre os séculos IV e V d.C., construída sob possíveis influências de Hiparco (BERLINGHOFF, GOUVÊA, 2012). Tanto os indianos quanto os árabes proporcionaram o fortalecimento da Trigonometria, pois diferentemente das aplicações das cordas apresentadas por Ptolomeu, os indianos utilizaram a relação entre metade de uma corda de um círculo e a metade do ângulo central correspondente, relação está conhecida como jya e presente no texto de aproximadamente 400 d.C., denominado "Surya Siddhanta" (Sistemas do Sol), obra importante para a história da Trigonometria (SAMPAIO, 2008; SOUZA, 2013). O seno surgiu a partir dos escritos dessa obra (KENNEDY, 1992).

Iya é uma das várias grafias para a palavra "corda" em hindu. Matemáticos árabes transliteraram jya para $j y b$, incorretamente lida como jayb. Posteriormente, do árabe para o latim, o tradutor Gerardo de Cremona (1114 - 1187) traduziu para "sinus", atualmente denominada seno (KENNEDY, 1992). 
Segundo Kennedy (1992), o seno indiano ou meia-corda era definida como metade da corda dividida pelo raio do círculo, conforme representado na Figura 3:

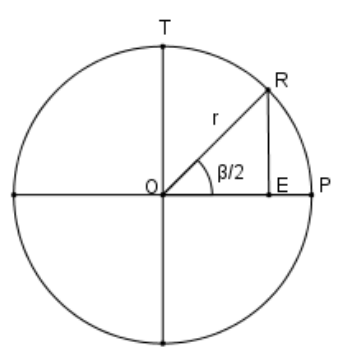

Figura 3 - Representação da meia-corda hindu.

Fonte: Adaptado de Kennedy (1992).

Matematicamente, obtém-se:

$\operatorname{sen}\left(\frac{\beta}{2}\right)=\frac{R E}{O R}=\frac{j y a}{r}=\frac{\frac{c r d \beta}{2}}{r}=\frac{\operatorname{crd} \beta}{2 r}$

Ao ter conhecimento a respeito do comprimento de uma corda por meio dos escritos gregos, aplicando-a na relação apresentada para o seno, obtém-se o valor da metade do arco. Como visto anteriormente, o $\mathrm{crd} 90^{\circ}=r \sqrt{2}$, então é possível encontrar o valor para a metade do arco:

$$
\operatorname{sen} 45^{\circ}=\operatorname{sen}\left(\frac{90^{\circ}}{2}\right)=\frac{\operatorname{crd} \beta}{2 r}=\frac{\operatorname{crd} 90^{\circ}}{2 r}=\frac{r \sqrt{2}}{2 r}=\frac{\sqrt{2}}{2}
$$

Note que a partir do uso da meia-corda é possível obter uma primeira visão de triângulo retângulo no círculo e sua relação com o seno. Entretanto, vale ressaltar que os valores das tabelas de seno variavam de acordo com o valor que era atribuído para o raio, visto que o valor do raio até então não era uma constante dentre os matemáticos da época. Estudos baseados em Ptolomeu, por exemplo, consideravam raio igual a $60 \mathrm{e}$ estudos baseados em Hiparco (como no caso dos indianos) consideravam o raio igual a 3438 (BERLINGHOFF, GOUVÊA, 2012).

Aryabhata (476 - 550 d.C.) utilizou do raio de 3438 para construir uma tabela de senos. Com isto, para cada arco a tabela dava o comprimento do seno do arco (KATZ, 2010). Berlinghoff e Gouvêa (2012) relatam que para se calcular raios diferentes a estes valores apresentados, usava-se de proporcionalidade para ajustar valores.

Morey destaca (2003. p. 19 e 20):

[...] para os indianos, as funções trigonométricas ainda eram definidas como comprimento de um segmento e não como uma relação entre dois comprimentos, como é o caso das funções trigonométricas modernas. Deste modo, quando dizemos seno indiano estamos nos referindo ao comprimento da meia-corda correspondente ao ângulo central. 
Em consequência do seno, tem-se o seno complementar, denominado pelos hindus como Kojya e representado na Figura 3 pelo arco que une os pontos $R$ e $T$. Berlinghoff e Gouvêa (2012) destacam que eventualmente a aplicação do seno complementar era necessária. Entretanto, até aquele momento da história, o conceito do mesmo não havia sido definido. Somente séculos depois com os estudos de Regiomontanus (1436 - 1475) o seno complementar foi conceituado (PEREIRA, MOREY 2015). Atualmente o seno complementar é denominado cosseno.

\section{Círculo de raio unitário}

Após o período de contribuição dos povos hindus, os árabes assumiram a Trigonometria das tabelas indianas de seno e proporcionaram relevantes contribuições para a Trigonometria. além de calcular o seno, os árabes calcularam as demais relações trigonométricas (cosseno, tangente, cotangente, secante, cossecante) (KATZ, 2010), sendo estas abordadas em seus respectivos tópicos nesta reconstruçãoUm marco da influência da civilização árabe foi à fundação da Escola de Bagdad no século IX d.C., tendo como um de seus maiores pensadores o matemático Al-Battani (855 - 929 d.C.) com seus trabalhos em Astronomia e a construção de diversas tabelas das linhas trigonométricas, tendo como base a Trigonometria indiana (SAMPAIO, 2008; BERLINGHOFF, GOUVÊA, 2012).

Segundo Costa (1997), Al-Battani foi quem introduziu o círculo de raio unitário e assim demonstrou que o seno é válido para qualquer triângulo retângulo, seja qual for o valor atribuído à hipotenusa e ao cateto oposto, conforme representado na Figura 4 . Seu interesse estava em calcular altitude do Sol, então, utilizou-se das razões trigonométricas e construiu tabelas mais precisas que as existentes na época.

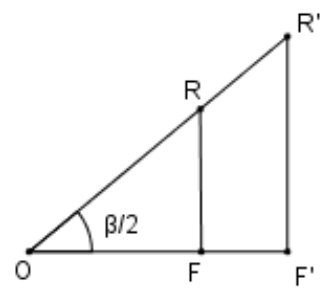

Figura 4 - Representação de seno para qualquer triângulo retângulo.

Fonte: Adaptado de Costa (1997).

Matematicamente, temos:

$$
\operatorname{sen}\left(\frac{\beta}{2}\right)=\frac{j y a}{1}=\frac{R F}{O R}=\frac{R^{\prime} F^{\prime}}{0 R^{\prime}}
$$

Com a pretensão de aperfeiçoar o Almagesto, Al-Battani usou do seno complementar em uma de suas obras astronômicas. Além disso, também ficou conhecido por utilizar de Álgebra ao invés de usar exclusivamente Geometria para os cálculos das tabelas de cordas (KATZ, 2010). Segundo Oliveira (2014), a articulação da Trigonometria de arcos e cordas com a Trigonometria de razões de triângulo retângulos só foi possível com a adoção do raio unitário como unidade de medida, pois até então era considerado 
por diversos matemáticos diferentes medidas de raio, ocasionando diferentes valores para o seno (GOMES, 2011).

\section{Seno e cosseno no triângulo retângulo}

Os estudos árabes foram disseminados na Europa, de modo que os Matemáticos europeus se familiarizaram com a Matemática árabe. Consequentemente, vários estudiosos europeus procuraram tornar mais precisas às tabelas de senos. Um desses estudiosos foi Nicolau Copérnico (1473 - 1543), cuja precisão de seus cálculos contribuiu para que sua tabela substituísse a tabela de Ptolomeu utilizada por muitos anos como modelo na Astronomia. Com estudos de Regiomontanus (1436 - 1475), as tabelas sexagesimais passaram a ser substituídas por tabelas decimais e a partir do seno conceituou-se o cosseno (SAMPAIO, 2008).

A obra de Regiomontanus intitulada "De triangulis Omnimodis Libri Quinque" (Triângulos de todos os tipos) é um trabalho dedicado a Trigonometria plana e esférica, dividida em cinco livros, dois dedicados à Trigonometria plana e três dedicados à Trigonometria esférica. Esta obra foi um marco histórico, pois até então a Trigonometria era estudada como parte da astronomia, mas a partir do século XVI se tornou independente. Regiomontanus trabalhou conceito de seno e cosseno, os quais diferem muito pouco do que se considera atualmente.

Pereira e Morey (2015) apresentam o teorema referente ao seno, presente no primeiro livro da obra de Regiomontanus:

Teorema 20: Em todo triângulo retângulo, um dos quais o vértice agudo é o centro de um círculo e cuja hipotenusa é o raio, o lado que subtende a este ângulo agudo é o seno reto do arco adjacente ao lado oposto ao ângulo dado e o terceiro lado do triângulo é igual ao seno do complemento do arco (PEREIRA, MOREY 2015, p. 150).

A fórmula que Regiomontanus usava para representar o valor do seno complementar de um ângulo era $\operatorname{sen}\left(90^{\circ}-\theta\right)$, ou seja, a subtração entre o ângulo de noventa graus e o valor do ângulo do seno (BERLINGHOFF, GOUVÊA, 2012).

Sobre o termo seno complementar, Edmund Gunter (1581 - 1626) associou "complemento" com "seno", tornando-se "co-sinus", logo passou para "cosinus", e no português denominou-se "co-seno" ou "cosseno" (KENNEDY, 1992).

Outro estudioso europeu que contribuiu com o desenvolvimento da Trigonometria foi Rheticus (1514 - 1576), que se dedicou ao estudo de triângulos retângulos e definiu as relações com ângulos ao invés de arcos, ou seja, quando se referia ao seno, não o representava mais por seno de um arco, mas sim por seno de um ângulo. Esta contribuição corresponde a um marco na História por proporcionar um elevado grau de desenvolvimento na Trigonometria (GOMES, 2011; SAMPAIO, 2008; KLINE, 1972).

Sampaio (2008) cita a definição que Rheticus utilizou para definir seno e cosseno no triângulo retângulo: "Em todo o triângulo com ângulo reto, o lado que subtende ao ângulo reto é chamado de hipotenusa. Se $\overline{A B}$ é o raio ou "sinus totus" (seno total), então a perpendicular é o seno e a base é o cosseno" (ZELLER, 1944, p. 56 apud SAMPAIO, 
2008), como mostra a Figura 5. Consequentemente, Rheticus definiu as relações trigonométricas por razões entre lados de um triângulo retângulo utilizando de termos "perpendiculum" (perpendicular), "basis" (base) e "hypotenusa" (hipotenusa) (OLIVEIRA, 2013).

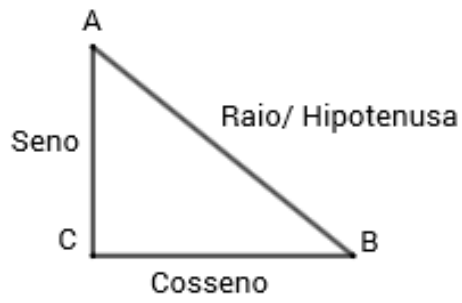

Figura 5 - Componentes de um triângulo retângulo.

Fonte: Os autores (2018).

Definição de função seno e função cosseno

Diante da sistematização do conhecimento trigonométrico e sua representação em triângulos retângulos, os europeus desenvolveram as Funções Trigonométricas. Tal desenvolvimento foi possível devido aos estudos sobre a Geometria Analítica.

Geometria Analítica, também conhecida como Geometria das coordenadas surgiu em 1637 por René Descartes (1596 - 1650) e Pierre de Fermat (1601 - 1665). Ambos apresentaram em seus estudos técnicas básicas para relacionar e Álgebra e a Geometria, as quais, posteriormente, com as contribuições de John Wallis (1616 - 1703), Isaac Newton (1642 - 1727) e outros matemáticos, resultaram na Geometria Analítica conhecida atualmente (KATZ, 2010; KLINE, 1972).

A Álgebra simbólica abriu caminhos para a Geometria Analítica, que por sua vez, abriu caminhos para as Funções Trigonométricas, sobretudo a partir de trabalhos desenvolvidos pelo matemático Leonhard Euler (1707 - 1783) (BERLINGHOFF, GOUVÊA, 2012).

Berlinghoff e Gouvêa (2012) descrevem Euler como "uma mente com superabundância de ideias". Ele se dedicou a diversos ramos da Matemática e da Física, além da Astronomia, Engenharia e Filosofia. A História revela que Euler teve grande contribuição no desenvolvimento da Matemática. Na Trigonometria em particular, contribuiu com estudos sobre Funções Trigonométricas. Ele trabalhou com uma Matemática simbólica, das quais destaca-se: $f(x)$ para representar funções; $a, b, c$ para representar os lados de um triângulo $A B C$; para as funções seno e cosseno usou de abreviações como sen e cos; entre outras contribuições (BOYER, 2012).

Além disso, Euler trabalhou com funções no sentido moderno da palavra, ou seja, representada por expressões analíticas. A partir de seus trabalhos estabeleceu-se a Trigonometria conhecida atualmente. Euler trabalhou em estudos com a unidade de medida em radianos, na qual o comprimento de um arco de raio unitário mede exatamente uma unidade (BERLINGHOFF, GOUVÊA, 2012; GOMES, 2011), conforme se observa na Figura 6. 


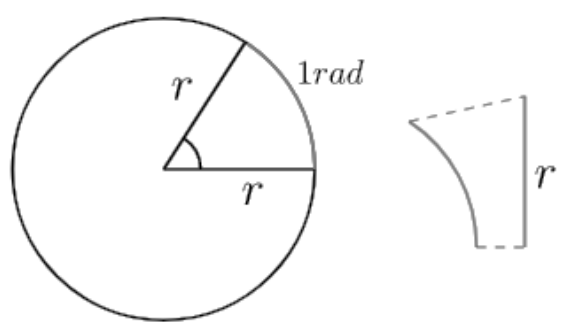

Figura 6 - Representação dos radianos como medida angular.

Fonte: http://www.matika.com.br/radianos/definicao-do-radiano

Quintaneiro, Giraldo e Pinto (2010) destacam que a única maneira de atender simultaneamente a necessidade da Trigonometria da circunferência e da Trigonometria do triângulo retângulo é com raio sendo uma unidade de medida única, ou seja, Euler adotou o radiano ${ }^{1}$ como unidade de medida, sendo definido como medida angular e medida linear. Desse modo, o radiano passou a ser usado como unidade padrão no estudo das Funções Trigonométricas do círculo/circunferência trigonométrica (OLIVEIRA, 2014).

A partir de estudos com radiano, Euler considerou um círculo $C$ com centro em $(0,0)$ e raio unitário. Fixando o círculo no ponto $A(1,0)$, que representa a origem dos arcos, associou cada número como um ponto do círculo $C$. Assim, criou a função Euler $(E)^{2}$. O domínio da função $E$ é o conjunto dos números reais $(\mathrm{R})$ e o contradomínio é $C$. Portanto, a função $E: \mathbb{R} \rightarrow C$ associa cada $x \in \mathbb{R}$ um ponto $P \in C, P=(a, b)$ pertence a $C$, se e somente se $a^{2}+b^{2}=1$ (COSTA, 1997).

No livro "Introductio in Analysin Infinitorum" de 1748, Euler realizou o tratamento Analítico das Funções Trigonométricas, considerado este a obra chave da Análise Matemática. A partir da função $E$, sendo $E(\theta)=(\cos (\theta), \operatorname{sen}(\theta))$, define que $\cos (\theta)=x \mathrm{e}$ $\operatorname{sen}(\theta)=y$, ou seja, definiu seno e cosseno como um número obtido pelas coordenadas, não mais como segmento de reta, de modo que o seno corresponde a coordenada $y$ (ordenada) e o cosseno como coordenada $x$ (abscissa) em um círculo unitário (OLIVEIRA, 2013), conforme pode-se observar na Figura 7.

10 termo radiano (radian) foi impresso pela primeira vez somente em (1873) por James Thonson, provavelmente inspirado pela palavra "radius" (raio) (QUINTANEIRO, GIRAL, PINTO, 2010).

${ }^{2}$ Para maior conhecimento sobre a função Euler, Oliveira (2014) aborda detalhadamente seu desenvolvimento, a partir da qual a Trigonometria passou a ser trabalhada em uma circunferência trigonométrica, consequentemente, levando os matemáticos a estudarem seus comportamentos e esboçando-as como funções (OLIVEIRA, 2014). 


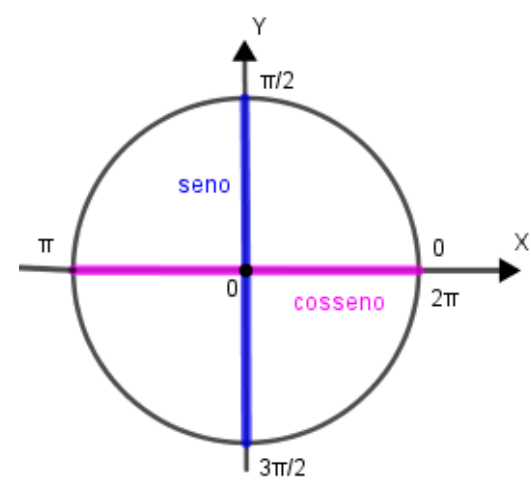

Figura 7 - Representação de seno e cosseno na circunferência trigonométrica.

Fonte: Adaptado de Oliveira (2013).

Euler considerou a função $E$ periódica ao realizar uma volta completa no círculo $C$, ou seja, $2 \pi$, e a partir de então definiu as funções seno e cosseno como periódicas em $2 \pi$.

As funções periódicas possuem aplicação em diversas áreas, como:

[...] música (a teoria da ressonância afirma a natureza matemática nas relações harmônicas), acústica (no estudo dos meios de propagação do som), eletricidade (no estudo do eletromagnetismo, equações matemáticas preveem ondas eletromagnéticas), mecânica (no movimento circular uniforme), entre outros (OLIVEIRA, 2013, p. 64-65).

Em relação à representação gráfico da função seno, no século XVII, Gilles de Roberval (1602 - 1675) fez o primeiro esboço de uma curva seno ao calcular a área sob uma cicloide. Mas o entendimento claro de como isso pôde ser feito foi detalhado a partir dos estudos de Euler (BERLINGHOFF, GOUVÊA, 2012). As relações trigonométricas, seno e cosseno, que inicialmente estavam ligadas a cálculos astronômicos, passaram a ser tratada com elementos da Geometria Analítica. Essa nova articulação proporcionou à Trigonometria um grande avanço em seu desenvolvimento e contribuir para que se tornasse um dos estudos independente da Matemática, com aplicações em diversas áreas do conhecimento.

\section{O desenvolvimento das relações tangente, cotangente, secante e cossecante}

A origem das relações tangente, cotangente, secante e cossecante diferem da origem do seno e cosseno, pois foram definidas a partir de sombras projetadas por um gnômon (Relógio do Sol). Antigas civilizações já conheciam essas relações e em um determinado período estudiosos árabes também as consideraram.

\section{Inclinação da pirâmide}

A História relata que os egípcios são considerados predecessores das funções tangente e cotangente, as quais tiveram seu desenvolvimento de forma concomitantemente, mas não devido à estudos astronômicos. Suas origens estão diretamente ligadas a estudos de alturas e distâncias. 
De acordo com o papiro Rhind, um dos documentos antigos mais importantes do Egito, diante da necessidade de manter uma inclinação constante das faces das pirâmides, os egípcios introduziram o conceito de "seqt", que corresponde a razão entre afastamento horizontal e elevação vertical (BOYER, 2012; EVES, 2011). A Figura 8 representa uma pirâmide de base quadrada, como as do Egito, na qual destaca-se a razão entre o afastamento horizontal e a elevação vertical: o seqt.
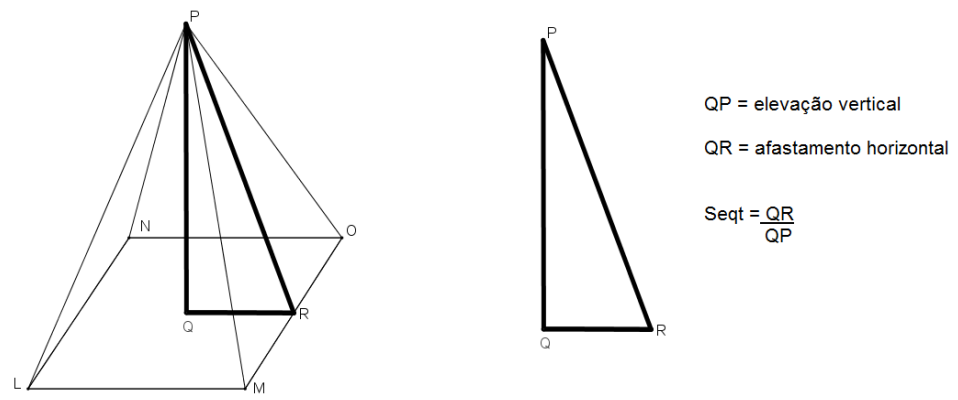

Figura 8 - Demonstração do seqt em uma pirâmide.

Fonte: Adaptado de Costa (1997).

Atualmente, o conceito de seqt é equivalente à cotangente de um ângulo, como Boyer (2012) e Eves (2011) afirmam, e pode ser representado matematicamente por:

$$
\text { seqt }=\text { cotangente } \theta=\frac{Q R}{Q P}=\frac{\cos \theta}{\operatorname{sen} \theta}
$$

Projeção de sombras do gnômon

A aplicabilidade das relações tangente e cotangente pelos egípcios evidencia-se a partir das sombras projetadas por um gnômon, objeto constituído de uma vareta de comprimento definido espetada perpendicularmente no chão, a partir da qual se observava o comprimento de sua sombra. Portanto, possivelmente os egípcios utilizavam o conhecimento de ângulos relacionado ao comprimento da sombra ao longo do dia, e assim calculavam tabelas de sombras (COSTA, 1997; KENNEDY, 1992).

Os árabes, séculos depois, também se dedicaram as tabelas de sombras utilizando do gnômon, tanto para sombras verticais como horizontais e assim trabalharam com tangente, cotangente, secante e cossecante (KENNEDY, 1992).

O matemático árabe Abul-Wêfa (940 - 998) introduziu a secante e cossecante e calculou tabela de tangente. Contudo, alguns anos antes, Habas (770 - 870) escreveu uma obra contendo as relações tangente, cotangente, secante e cossecante. Al-Biruni (973 - 1055) no seu Tratado Exaustivo sobre as Sombras apresenta de uma forma mais clara essas relações vinculadas a sombras de um gnômon (KATZ, 2010).

De acordo com o historiador Katz (2010), tendo um gnômon representado por $\overline{A B}$ (perpendicular ao plano), conforme representado na Figura 9, a sombra do gnômon (ou "sombra direta") representado por $\overline{B C}$ (paralelo ao plano) foi denominada cotangente e o raio de incidência do sol (linha que une as duas extremidades da sombra e do gnômon) representado por $\overline{A C}$ (hipotenusa da sombra), denominado de cossecante, de acordo com o ângulo $\theta$. 


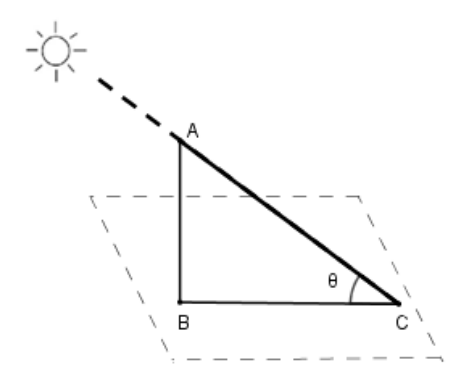

Figura 9 - Representação de cotangente e cossecante pela definição de Al-Biruni.

Fonte: Adaptado de Katz (2010).

Por outro lado, considerando o gnômon paralelo ao plano horizontal, ou seja, perpendicular ao plano apresentado na Figura 10 e representado por $\overline{A B}$, tem-se que $\overline{A C}$ constitui o raio do sol e $\overline{B C}$ a sombra do gnômon. A partir dessas definições, a "sombra invertida" $\overline{B C}$ foi estabelecida como tangente e $\overline{A C}$ (hipotenusa da sombra invertida) como secante, a partir do ângulo $\beta$.

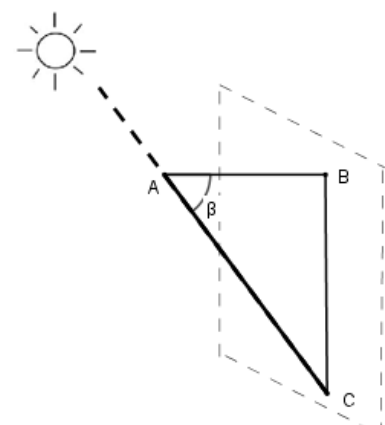

Figura 10 - Representação de tangente e secante pela definição de Al-Biruni.

Fonte: Adaptado de Katz (2010).

Para atribuir valores às relações tangente e cotangente, Al-Biruni elaborou uma tabela, na qual considerou a tangente como quociente de seno e cosseno. Por outro lado, para a cotangente utilizou-se da relação $\cot \theta=\operatorname{tg}(90 \varrho-\theta)$, ou seja, ele considerava a cotangente complementar a tangente (KATZ, 2010). De qualquer forma, vale ressaltar que, de acordo com o apresentado anteriormente, os egípcios já possuíam conhecimento a respeito da cotangente, atualmente definida pelo quociente de cosseno e seno.

Dentre as razões que Al-Biruni apresentou, ele mostrou a razão cossecante da seguinte forma: estabeleceu razão entre o comprimento do gnômon $(g)$ pela hipotenusa da sombra $(g \operatorname{cossec} \theta)$ e igualou com a razão entre o seno da altitude $(r \operatorname{sen} \theta)$ pelo seno total traduzida como (KATZ, 2010):

$$
\frac{g}{g \cos \sec \theta}=\frac{r \operatorname{sen} \theta}{r}
$$

Considerando que o raio possui valor unitário e o seno total equivale ao valor do raio, por meio do Teorema de Tales pode se estabelecer a razão da $\operatorname{cossec}(\theta)$ como: 


$$
\begin{aligned}
& \frac{1}{\operatorname{cossec} \theta}=\frac{\operatorname{sen} \theta}{1} \\
& \operatorname{cossec} \theta=\frac{1}{\operatorname{sen} \theta}
\end{aligned}
$$

De forma análoga, pode-se atribuir uma relação para a secante. De acordo com as demonstrações realizadas, tanto a cossecante quanto a secante são consideradas hipotenusas, logo, tem-se que:

$$
\sec \theta=\frac{1}{\cos \theta}
$$

Os significados atribuídos atualmente para as relações trigonométricas se tornam claros a partir da interpretação geométrica, considerando um círculo de raio unitário e um ângulo central. Segundo Eves (2011), a partir da Figura 11, pode-se definir:

Se o raio do círculo é uma unidade, os valores de $\operatorname{tg} \theta$ e $\sec \theta$ são dados pelos comprimentos do segmento de tangente $C D$ e pelo segmento de secante $O D$, respectivamente. Obviamente, cotangente significa simplesmente "tangente do complemento" e assim por diante. As funções tangente, cotangente, secante e cossecante foram conhecidas por vários outros nomes, sendo definidas os nomes atuais até o fim do século XVI (EVES, 2011, p. 266).

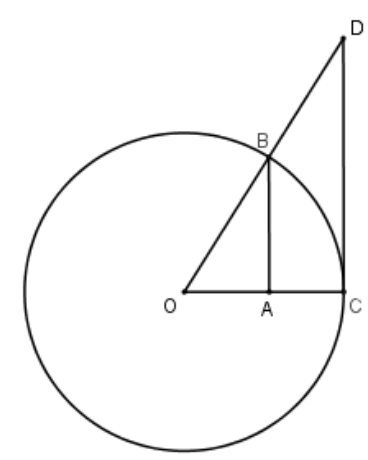

Figura 11 - Relações trigonométricas no círculo unitário.

Fonte: Adaptado de Eves (2011).

Contudo, de acordo com Sampaio (2008), as contribuições de Al-Biruni foram utilizadas apenas para usos astronômicos. No decorrer da História, outros matemáticos se dedicaram a calcular tabelas a respeito dessas relações e aprimorá-las. Rheticus, além de trabalhar com seno e cosseno, também trabalhou com tangente, cotangente, secante e cossecante, definindo-as como razões relacionando ângulos de triângulo retângulo. Euler, assim como fez com seno e cosseno, estabeleceu essas outras quatro relações como funções, ampliando para seis as Funções Trigonométricas, atribuindo-Ihes as abreviações utilizadas atualmente: tang, cot, sec, cosec (SAMPAIO, 2008). Porém, não foi encontrado fontes históricas que demonstrassem como essas funções foram definidas por Euler. Ademais, vale ressaltar que em 1759 as funções passaram a ser definidas por números que representam ângulos, tornando-se $\operatorname{sen} x, \cos x, \operatorname{tg} x, \operatorname{cotg} x, \sec x, \operatorname{cossec} x$. Atualmente, 
a Trigonometria é concebida como um conjunto de números complexos, não sendo necessário recorrer a arcos ou ângulos (KENNEDY, 1992).

\section{Considerações Finais}

Fundamentado em referências como Eves (2011), Boyer (2012), Sampaio (2008), Costa (1997), Katz (2010), Kline (1972), Berlinghoff e Gouvêa (2012), foi possível a realização de uma síntese histórico-epistemológica das Funções Trigonométricas. Sua sistematização foi cunhada por diversas civilizações em diferentes períodos, estando intimamente ligada a um contexto prático que, posteriormente, se tornou uma área de conhecimento específica, na qual pôde abranger diversas áreas de aplicação. O caminho percorrido para que se reconstruísse esse conhecimento nos mostra o quanto a Matemática está intimamente ligada ao cotidiano das civilizações, tendo em vista sua aplicabilidade.

A História da Matemática nos proporciona um olhar que vai além da Matemática pronta que muitas vezes nos é apresentada. Além disso, quando associada à epistemologia, podemos compreender a construção de conceitos e estruturas, no caso, atinentes à Trigonometria, proporcionando-Ihe, assim, significados.

Por fim, considera-se esta pesquisa relevante para pesquisadores e professores da área de estudo da Matemática, podendo se estender também a comunidade cientifica que queira aprofundar seus conhecimentos a respeito do desenvolvimento histórico e epistemológico das Funções Trigonométricas.

\section{Referências}

ALVES, Marcos Alexandre. TATSCH, Karla Jaqueline Souza. Epistemologia, História e Ensino de Matemática: Reflexões sobre formação e aprendizagem significativa. Revista de Ensino de Ciências e de Matemática. Cruzeiro do Sul, v. 8, n.3, p. 78-93, 2017.

BERLINGHOFF, William P.; GOUVÊA, Fernando Q. A Matemática através dos tempos: um guia fácil e prático para professores e entusiastas. 2 ed. São Paulo: Blucher, 2012.

BOYER, Carl B. História da Matemática. Tradução Helena Castro. 3. ed. São Paulo: Blucher, 504 f., 2012.

COSTA, Nielce Meneguel Lobo da. Funções seno e cosseno: uma sequência de ensino a partir dos contextos do "mundo experimental" e do computador. 1997. 250 f. Dissertação (Ensino da Matemática) - Pontifica Universidade Católica de São Paulo, São Paulo, 1997.

D’AMBROSIO, U. A História da Matemática: questões historiográficas e políticas e reflexos na Educação Matemática. In: BICUDO, M. A. V. (Ed.). Pesquisa em Educação Matemática: concepções e perspectivas. São Paulo: Editora UNESP, p. 97-115, 1999.

EVES, Howard. Introdução à história da Matemática. Tradução Hygino H. Domingues. 5. ed. Campinas: Unicamp, 843 f., 2011. 
GOMES, Severino Carlos. Elaboração e aplicação de uma sequência de atividades para o ensino de Trigonometria numa abordagem histórica. 2011. 90 f. Dissertação (Ensino de Ciências naturais e Matemática) - Universidade Federal do Rio Grande do Norte, Natal, 2011.

KATZ, Victor J. História da Matemática. 2. ed. Lisboa: Fundação Calouste Gulbenkian, 2010.

KENNEDY, Edward. Tópicos de História da Matemática para uso em sala de aula. São Paulo: Atual, 48 p., 1992.

KLINE, Morris. Mathematical Thought from Ancient to Modern Times. New York: Oxford University Press. 1972.

LOPES, Lidiane Schimitz; ALVES, Antônio Maurício Medeiros. A História da Matemática em sala de aula: propostas de atividades para a Educação Básica. Encontro Regional de Estudantes de Matemática Da Região Sul, p. 320-330, nov. 2014.

LOPES, Lidiane Schimitz; FERREIRA, André Luis Andrejew. Um olhar sobre a história nas aulas de matemática. Abakós, v. 2, n. 1, p. 75-88, nov. 2013.

LUCAS, Lucken Bueno. Contribuições axiológicas e epistemológicas ao ensino da teoria da evolução de Darwin. 2010. 170 f. Dissertação (Pós-graduação em Ensino de Ciências e Educação Matemática) - Universidade Estadual de Londrina, Londrina, 2010.

MELO, Enaldo Vieira. FIREMAN, Elton Casado. Ensino e aprendizagem de funções trigonométricas por meio do software GeoGebra aliado à modelagem matemática. Revista de Ensino de Ciências e de Matemática. Cruzeiro do Sul, v. 7, n.5, p. 12-30, 2016.

MENDES, Iran Abreu. Ensino de trigonometria através de atividades históricas. 1997. 216 f. Dissertação (Mestrado em Educação Matemática) -Universidade Federal do Rio Grande do Norte, Natal, 1997.

MIGUEL, Antonio. Três estudos sobre história e educação Matemática. 1993. 361 f. Tese (Doutorado em Educação) - Universidade Estadual de Campinas, Faculdade de Educação Departamento de Metodologia de Ensino Campinas, 1993.

MOREY, Bernardete. Geometria e Trigonometria na índia e nos Países Árabes. Coleção História da Matemática, 2003.

NASCIMENTO, Alessandra Zeman do. Uma sequência de ensino para a construção de uma tabela trigonométrica. 2005. 228 f. Dissertação (Mestrado em Educação Matemática) - Pontifica Universidade Católica de São Paulo, São Paulo, 2005.

OLIVEIRA, Carlos André Carneiro de. Trigonometria: os radianos e as funções seno, cosseno e tangente. 2014. 77 f. Dissertação (Programa de Pós-Graduação em Matemática -CCT - UFCG) - Universidade Federal de Campina Grande, Centro de Ciências e Tecnologia, Campina Grande, 2014.

OLIVEIRA, Juliana Elvira Mendes de. A Trigonometria na Educação Básica com foco em sua evolução histórica e suas aplicações contemporâneas. 2013. 134 f. 
Dissertação (Programa de Pós-Graduação do mestrado profissional em Matemática em Rede Nacional) - Universidade Federal de Viçosa, Viçosa, 2013.

PEREIRA, Ana Carolina; MOREY, Bernadete Barbosa. Um ensaio sobre a história da trigonometria antes do século XV. Conexões - Ciência e tecnologia, Fortaleza, v. 9, n. 4, p. 143-152, 2015, dez. 2015.

QUINTANEIRO, WIlerson; GIRALDO, Victor; PINTO, Márcia Fusaro. De onde vem a unidade radiano e por que seu uso é necessário. In: Encontro Estadual de Educação Matemática do Rio de Janeiro (EEMAT), 2010, Rio de Janeiro. Anais... Rio de Janeiro: EEMAT, p. 1-11, 2010.

SAMPAIO, Helenara Regina. Uma abordagem histórico-filosófica na Educação Matemática: contribuições ao processo de aprendizagem de trigonometria no Ensino Médio. 2008. 190 f. Dissertação (Pós-Graduação em Ensino de Ciências e Educação Matemática) - Universidade Estadual de Londrina, Londrina, 2008.

SCOTT, J. F. A HISTORY OF MATHEMATICS: From Antiquity to the Beginning of the nineteenth century. London: Taylor \& Francis LTD, 1969.

SOUZA, Thuysa Schlichting de. Um Estudo da Extensão do seno, cosseno e tangente no Triângulo Retângulo para Funções de Domínio Real. 2013. 54 f. Trabalho de conclusão de curso (Licenciatura em Matemática) - Universidade Federal de Santa Catarina, Florianópolis, 2013.

Submissão: 04/05/2018

Aceite: 18/06/2018 